\title{
Texture Fusion for Batik Motif Retrieval System
}

\author{
Ida Nurhaida ${ }^{1}$, Hong Wei ${ }^{2}$, Remmy A. M. Zen ${ }^{3}$, Ruli Manurung ${ }^{4}$, Aniati M. Arymurthy ${ }^{5}$ \\ ${ }^{1}$ Computer Science Faculty, Universitas Mercu Buana, Indonesia \\ ${ }^{2}$ School of System Engineering, University of Reading, UK \\ ${ }^{3,4,5}$ Computer Science Faculty, Universitas Indonesia, Indonesia
}

\section{Article Info}

Article history:

Received Aug 2, 2016

Revised Nov 8, 2016

Accepted Nov 22, 2016

\section{Keyword:}

Batik

Batik motif retrieval system

Content based image retrieval

Feature fusion

Motif

\begin{abstract}
This paper systematically investigates the effect of image texture features on batik motif retrieval performance. The retrieval process uses a query motif image to find matching motif images in a database. In this study, feature fusion of various image texture features such as Gabor, Log-Gabor, Grey Level Co-Occurrence Matrices (GLCM), and Local Binary Pattern (LBP) features are attempted in motif image retrieval. With regards to performance evaluation, both individual features and fused feature sets are applied. Experimental results show that optimal feature fusion outperforms individual features in batik motif retrieval. Among the individual features tested, LogGabor features provide the best result. The proposed approach is best used in a scenario where a query image containing multiple basic motif objects is applied to a dataset in which retrieved images also contain multiple motif objects. The retrieval rate achieves $84.54 \%$ for the rank 3 precision when the feature space is fused with Gabor, GLCM and Log-Gabor features. The investigation also shows that the proposed method does not work well for a retrieval scenario where the query image contains multiple basic motif objects being applied to a dataset in which the retrieved images only contain one basic motif object.
\end{abstract}

Copyright $\odot 2016$ Institute of Advanced Engineering and Science. All rights reserved.

\section{Corresponding Author:}

Ida Nurhaida, Computer Science Faculty, Universitas Mercu Buana, Jalan Raya Meruya Selatan no. 1, Kembangan Jakarta Barat-16550, Indonesia. Email: ida.nurhaida@mercubuana.ac.id

\section{INTRODUCTION}

Batik is fabric ornamented with figures of a specific natures. Batik is a famous cultural heritage from Indonesia, originally reserved as an art form for Javanese royalty. The word batik originates from the Javanese tik and means to dot. Batik is a method of dyeing designs on cloth by coating the parts that are not to be dyed with removable wax [1-2]. The patterns are drawn using a spoon-like tool (canting), stamping block, or brush. Bees wax (malam) patterns are applied to fabric during an immersion process. The parts covered in wax resist the dye and remain in the original colour. The wax is removed after the dyeing process. The ornamentations on a piece of batik arranged so as a total composition called motif [3]. The waxing and dyeing process can be repeated to create more motifs and designs.

Batik plays a meaningful role in Indonesia's cultural heritage. Batik gives significant contribution to global art as well. The philosophy of batik is related to the cultural identity of the Indonesian people and the symbolic meanings that express their creativity and spirituality. While various other countries also have their own varieties of batik, UNESCO has designated Indonesian batik as a Masterpiece of Oral and Intangible Heritage of Humanity in 2009. Indonesia's Batik patterns are classified as geometric patterns and non-geometric patterns. There are several groups of geometric patterns namely ceplok, kawung, parang, lereng, and nitik, while variety designs of non-geometric patterns consist of four classes namely Lung-lungan 
and Semen, Pagersari, Taplak Meja and Wayang. Each group have its own variations and distinctive features. Samples of real images batik motifs from geometric patterns can be seen in Figure 1.

The craft of batik must be conserved and preserved. To that end, our main goal is the documentation of batik in a large digital repository system. Batik Motif Retrieval System (BMRS) will retrieve images which are visually similar to the query image in a database. BMRS systems perform feature extraction as a preprocessing step. To retrieve specific batik motifs from this repository, a user can place a query to the system, which then automatically provides related information. Processing of query image involves extraction of image features and search in the visual feature space for similar images. The Top- $N$ most similar batik motif images are retrieved and presented to the user. BMRS systems perform feature extraction as a preprocessing step. Once obtained, image features act as inputs to subsequent image analysis tasks as similarity estimation. However, content-based retrieval systems have limitations between the human representation of an image and the low level features stored in the database, often called the Semantic Gap [4-6]. The reduction of the semantic gap and how to achieve accurate retrieval results are a challenging problem in Content Based Image Retrieval (CBIR) systems. The key problem in a batik motif retrieval system is the nature of object in batik motif, which consists of geometric ornaments with circle shape like flower or star, ellipse, and series of such objects in the form of dots and small lines in parallel position. The ornaments are placed in decorative area with variations in position, scale, and rotation. It is our hypothesis that these features complement each other in representing the batik motif properties in an image format. Experimental results have shown that the fusion of features extracted by different methods increases recognition rate in the batik motif retrieval system.

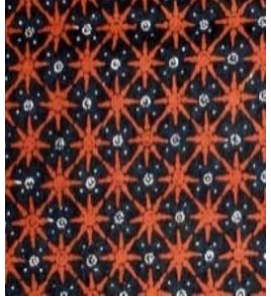

(a) Truntum Sogan of Ceplok class

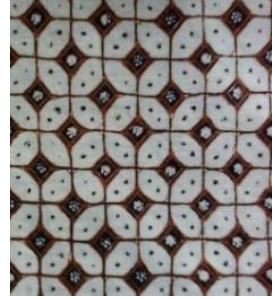

(b) Kawung Picis of Kawung class

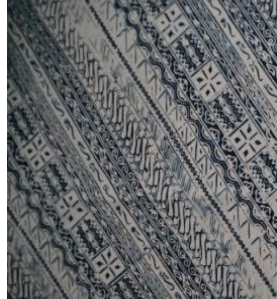

(c) Lereng Udan Liris of Lereng class

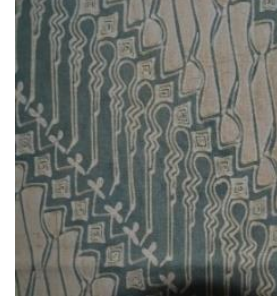

(d) Parang Rusak of Parang class

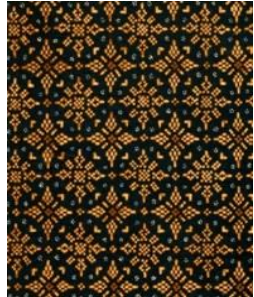

(e) Nitik Randu of Nitik class

Figure 1. Batik motif from geometric patterns. Ceplok motif has repetitive geometric ornaments based on circular shapes, stars, squares, cubes and other geometric lines. Kawung motif is the oldest known batik pattern. Kawung motif consists of the repetition of circles or elliptical shapes that that touch or overlap. Lereng motif has diagonal rows of patterns in between filled with small patterns. Parang motif consists of some parallel lines in diagonal form filled with small ornaments. Nitik motif is created with small dots and dashes imitating the original woven fabric (Reference: Batik Museum Jakarta, Indonesia and Sakundria Collection).

This paper builds on earlier work [7] in which a comparison was conducted based on a single texture feature in the domain of a batik image database. It studied batik motif identification in comparing with other labeled batik motif in the database. The highest performance of classification accuracy achieved nearly $80 \%$ using Grey Level Co-occurrence Matrix features. Shape similarity and texture characteristics have also been employed for batik image retrieval [8]. This research utilized edge detection and shape invariant moment as a feature. A thresholding approach is used to retrieve the images based on the value of the highest-grade representation on each image query. The best performance achieved a precision and recall of $70 \%$ and $75 \%$ respectively. In another study of batik image retrieval, similar values of $74 \%$ and $89 \%$ were obtained [9]. This study applied edge feature orientation combined with micro structure descriptor for enhancing retrieval performance. Rangkuti, et al [10] reported using Canny edge detection to an input image, wavelets as texture features and invariant moment as shape features method. The performance results achieved optimal precision of average $90 \%-92 \%$.

In this article, we first show that combining multiple texture description methods significantly improves the performance compared to using the single texture method alone for Batik Motif Retrieval. The idea is that the theoretical properties of each feature are examined in order to select the robust and reliable features for fusion. We particularly focus on discussing and evaluating how the single feature and fusion 
features perform in the retrieval of batik motif. Finally, we provide a comprehensive evaluation of both multiple features and single feature for the task of image retrieval batik motif. This will contribute towards the development of a digital image repository system of batik motifs for cultural heritage preservation solutions.

In this paper, we conduct systematical analysis to investigate the influence of image texture feature fusion to the performance on a batik motif retrieval system. The paper is organized as follows. In Section 2, the algorithm of feature extraction methods, fusion method, and similarity distance are elaborated. In Section 3 , the performance of feature fusion using similarity distance is tested and compared in a series of experiments. Finally, the result of CBIR experiments are discussed in Section 4 and conclusions are given in Section 5.

\section{RESEARCH METHOD}

\subsection{Feature Extraction Methods and Performance Evaluation}

In this paper, we use various texture features, i.e. Gabor filters, Log Gabor filters, Grey Level Co-occurrence Matrices, and Local Binary Patterns; and analyze their combination in batik motif image retrieval applications. The spatial locality, orientation selectivity, and frequency are captured as the main characteristics for representation of salient visual properties [11-12].

\subsubsection{Gabor Filter}

Gabor filters are used to model the spatial summation properties of simple cell in the visual cortex [13-14]. Filtering operation is conducted by image convolution of an original image with a Gabor filter to generate a new image. The number of new images is correlated to the number of filters used. A 2D Gaussian envelop is modulated a 2D Gabor filter in complex sinusoidal wave. The 2D Gabor filters can be categorized into two components: a real part as symmetric component and an imaginary part as the asymmetric component. The 2D Gabor function can be mathematically formulated as [14]:

$$
G(x, y, f, \theta)=\frac{f^{2}}{\pi \gamma \eta} e^{-\left(\frac{f^{2}}{\gamma^{2}} x^{\prime 2}+\frac{f^{2}}{\eta^{2}} y^{\prime 2}\right) e^{i 2 \pi f x^{\prime}}}
$$

where :

$x^{\prime}=x \cos \theta+y \sin \theta$

$y^{\prime}=-x \sin \theta+y \cos \theta$

In equation (1), $f$ is the frequency of sinusoidal wave, $\theta$ represents the anti-clockwise rotation of the Gaussian envelope and the sinusoid, $\gamma$ denotes the smoothing parameters of the Gaussian envelope, and $\eta$ indicates the orthogonal to the direction of the wave, respectively.

The total number of frequencies $n_{f}$ and the total number of orientations $n_{o}$ of the Gabor filters are determined to design Gabor filter bank. The combination of frequency and orientation generates the Gabor filter bank [13]. Research by Clausi [14] selected highest frequency $f_{m}=\sqrt{2} / 4$, four number of frequency $n_{f}(22.63,11.31,5.66$, and 2.83 pixel per cycle $)$ and six orientations $n_{o}\left(0^{\circ}, 30^{\circ}, 60^{\circ}, 90^{\circ}, 120^{\circ}\right.$, and $\left.150^{\circ}\right)$ to filter each test image. These filters are purposed to well-localized measure of the local information.

\subsubsection{Grey Level Co-occurrence Matrices}

Grey Level Co-occurrence Matrix (GLCM) is a common method used for analyzing image textures. The basic idea from this method is to extract homogeneous characteristics from image texture. GLCM produces features which describe well the relationship of adjacency among pixels in an texture image [15]. The second order statistics are accumulated into a set of 2D matrices, $P(i, j \mid d)$, each of which measures the spatial dependency of two gray levels, $\mathrm{i}$ and $\mathrm{j}$, given a displacement vector $\delta=(d, \theta)=(d x, d y)$ [13]. The number of occurrences (frequencies) of $\mathrm{i}$ and $\mathrm{j}$, separated by distance $\mathrm{d}$, contributes the $(\mathrm{i}, \mathrm{j}$ ) entry in the co-occurrence matrix $P(i, j \mid d)$. A co-occurrence matrix is given as:

$$
\begin{aligned}
& P(i, j \mid d)=\left\|\left\{\left(\left(x_{1}, y_{1}\right),\left(x_{2}, y_{2}\right)\right): \mathrm{I}\left(x_{1}, y_{1}\right)=i, \mathrm{I}\left(x_{2}, y_{2}\right)=j\right\}\right\| \\
& \left(x_{1}, y_{1}\right),\left(x_{2}, y_{2}\right) \epsilon w \times h,\left(x_{2}, y_{2}\right)=\left(x_{1} \pm d x, y_{1} \pm d y\right)
\end{aligned}
$$

where:

$P(i, j \mid d) \quad=$ Number of occurrences of the pair of grey levels $\mathrm{i}$ and $\mathrm{j}$

$\left(x_{1}, y_{1}\right)$ and $\left(x_{2}, y_{2}\right)=$ coordinates of pixels in two position 
$\delta \quad=$ displacement vector

$d \quad=$ distance

$\theta \quad=$ orientation

$\|\cdot\| \quad=$ Cardinality of a set

Texture features, such as homogeneity, energy, entropy, contrast, and correlation, are then derived from the co-occurrence matrix.

a. Contrast :

$$
\text { Contrast }=\sum_{i=1}^{k} \sum_{j=1}^{k}\left(P_{i, j}\right)(i-j)^{2}
$$

where:

$\mathrm{k}=$ the number of rows or columns

The probability of two pixels $P(i, j)$ indicated the separation of two pixels with different grey level $\mathrm{i}$ and $\mathrm{j}$

[6]. Contrast measures local intensity of invariance.

b. Homogeneity or Angular Second Moment (ASM) :

$$
A S M=\sum_{i=1}^{k} \sum_{j=1}^{k}\left(P_{i, j}\right)^{2}
$$

The homogeneity of an image is measured by using Angular Second Moment. The sum of squares will be high if homogeneous scene contains only a few gray levels but relatively high values of $P(i, j)$.

c. Inverse Difference Moment (IDM) :

$$
I D M=\sum_{i=1}^{k} \sum_{j=1}^{k} \frac{1}{1+(i-j)^{2}} P(i, j)
$$

The homogeneity of the image affected the IDM's value. IDM value will be low if the image is not homogeny. Otherwise, for homogeneous image, the IDM value is high.

Correlation

Correlation is a measure of gray level linear dependence between the pixels at the specified positions relative to each other.

$$
\text { Correlation }=\sum_{i=1}^{k} \sum_{j=1}^{k} P_{(i, j)} \frac{\left(i-\mu_{i}\right)\left(j-\mu_{j}\right)}{\sigma_{i} \sigma_{j}}
$$

where $\mu_{x}$ and $\mu_{y}$ are mean value of row $\mathrm{i}$ and column $\mathrm{j}$ and $\sigma_{x}$ and $\sigma_{y}$ are standard deviation of row $\mathrm{i}$ and column j.

\subsubsection{Log Gabor Filter}

Field proposed Log-Gabor filters as a modification to the basic Gabor function [16] (Field 1987). The singularity of the log function Log Gabor filters basically are defined in the frequency domain as Gaussian functions that shift from the origin [17]. Gabor filters present a limitation in the bandwidth where only bandwidth of 1 octave maximum could be designed [18-20]. Log Gabor consists of a logarithmic transformation in the Gabor domain which eliminates the DC-component allocated in medium and high-pass filters. The frequency response is a Gaussian on a log frequency axis. The comparison between Gabor and Log Gabor functions can be seen in Figure 2.

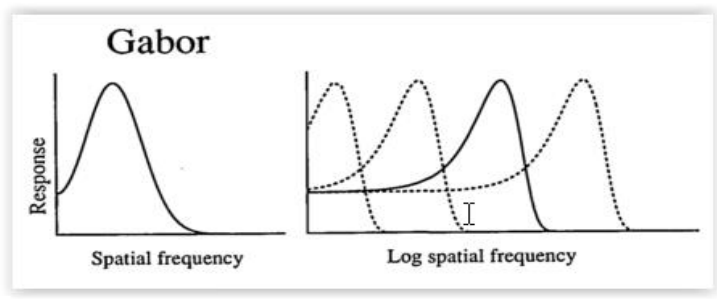

(a) Gabor Function

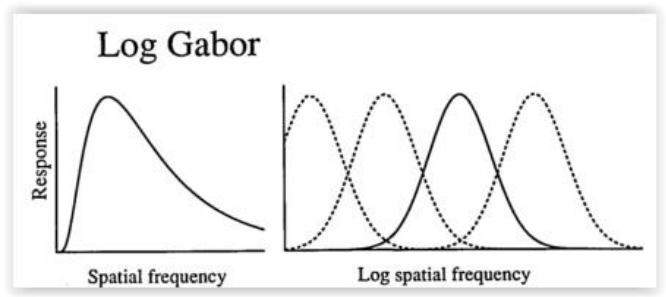

(b) Log Gabor Function

Figure 2. Comparison of Gabor and Log Gabor Functions [16] 
The frequency response of Log Gabor filter can be defined as:

$$
G(f)=\exp \left(-\left[\log \left(\frac{f}{f_{0}}\right)\right]^{2} / 2\left[\log \left(\frac{\sigma}{f_{0}}\right)\right]^{2}\right)
$$

where $f_{0}$ is a center frequency of a filter and $\sigma$ is a scaling factor of a radian bandwidth [16].

\subsubsection{Local Binary Pattern (LBP)}

The LBP operator is one of the best performing texture descriptors and it has been widely used in various applications. The complexity time will be minimized whether preprocessing and LBP are applying respectively [21]. The Local Binary Pattern (LBP) operator utilizes the center value as a reference in a $3 \times 3$ pixel neighborhood [22]. The threshold value is from the center pixel while the pixel value of a neighbor is marked as " 0 " when it is below the threshold and " 1 " otherwise. A binary number is formed to characterize the local texture (see Figure 3). Then, subtracting the average grey levels below the center pixel from the grey level above or equal to the center pixel will result in Contrast (C).

\begin{tabular}{|r|r|r|}
\hline 20 & 10 & 5 \\
\hline 7 & 10 & 12 \\
\hline 9 & 1 & 11 \\
\hline
\end{tabular}

(a) Example

\begin{tabular}{|l|l|l|}
\hline $1_{1}$ & $1_{2}$ & $0_{4}$ \\
\hline $0_{128}$ & & $1_{8}$ \\
\hline $0_{64}$ & $0_{32}$ & $1_{16}$ \\
\hline
\end{tabular}

(b) Thresholder

\begin{tabular}{|r|r|r|}
\hline 1 & 2 & 0 \\
\hline 0 & & 8 \\
\hline 0 & 0 & 16 \\
\hline
\end{tabular}

(c) Weights

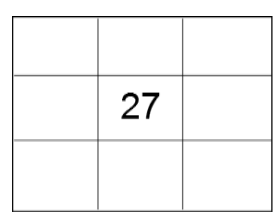

(d) LBP Calcu-lation

Figure 3. An Example of Local Binary Pattern Calculation

A binary number is represented as follow:

$$
L B P_{R, N}(x, y)=\sum_{i=0}^{N-1} s\left(n_{i}-n_{c}\right) 2^{i}, \quad s(x)=\left\{\begin{array}{cc}
1, & x \geq 0 \\
0 & \text { otherwise }
\end{array}\right.
$$

where:

$n_{c}=$ the gray level of the center pixel of a local neighborhood

$n_{i}=$ the gray levels of $\mathrm{N}$ evenly spaced pixels on a circle of radius $\mathrm{R}$.

\subsection{Feature Fusion}

Feature fusion integrates information from all available features into a unified representation [23]. Data fusion can be conducted at three distinct levels, i.e. feature level fusion, matching/score level, and decision level [24]. Feature level fusion is performed by concatenating the features resulting from feature extraction process. It is more complicated since a set of features probably have different dimension. However, feature level fusion has been considered preferable because fused features may contain additional distinct information to individual features. On the other hand, it is possible to select features for eliminating redundant information from a feature set.

Implementation for fusion at matching/score level is most frequently used compared to feature fusion in the lower level. Fusion at decision level gathers information after a decision is taken by a matcher based on its delivered input [25]. The final decision is made by a majority vote scheme, behavior knowledge space, weighted voting, and AND rule and OR rule. Fusion at the higher level may decrease the recognition performance since some information will be lost in the course of fusion process.

\subsection{Feature Matching}

Feature matching is a fundamental problem in computer vision, and plays a critical role in many tasks such as object recognition and localization [26]. A similarity measure for content-based retrieval should be efficient enough to match similar images as well as being able to discriminate dissimilar ones [6]. Feature vectors usually exist in a very high-dimensional space. The problem of matching can be defined as establishing a mapping between features in one image and similar features in another image. Similarity measure on this research was conducted using a Euclidian distance function. The Euclidean distance is a 
distance function most widely used to measure the distance of two vectors. If the two vectors are vectors $\mathrm{A}$ and $\mathrm{B}$, where:

$$
A=\left[\begin{array}{c}
a_{1} \\
a_{2} \\
a_{3} \\
\vdots \\
a_{n}
\end{array}\right] \text { and } B=\left[\begin{array}{c}
b_{1} \\
b_{2} \\
b_{3} \\
\vdots \\
b_{n}
\end{array}\right]
$$

The Euclidian Distance is defined as:

$$
\text { Euclidian Distance }=\sqrt{\sum_{i=1}^{n}\left(a_{i}-b_{i}\right)^{2}}
$$

\subsection{Performance Evaluation}

Precision and Recall are two indicators of the correctness retrieval result [27-28]. Recall means a ratio between the number of correctly retrieved image by the system and the number of all images in the database which have the same class with the query. In other words, recall describes the system's capability to retrieve relevant images, whereas precision is the number of relevant images found compared to the number of all images found in a query process. Precision depicts the ability of the system to reject the irrelevant image. A perfect precision score of 1.0 means that every result retrieved by a search was relevant whereas a perfect recall score of 1.0 means that all relevant images were retrieved by the search. Recall and precision are inversely related and can be defined as:

$$
\begin{aligned}
& \text { Precision }=\frac{\text { number of correct retrieved images }}{\text { total number of retrieved images }} \\
& \text { Recall }=\frac{\text { number of correct retrieved images }}{\text { the number of images representative of the query }}
\end{aligned}
$$

\subsection{Proposed Method}

In this paper, we propose a CBIR approach for batik motif retrieval system that builds on texture feature fusion through an effective selection feature. Furthermore, the proposed approach was extensively evaluated on the image data set of batik. It should be noted that we use the same parameter used by [7] for GLCM and Gabor feature extraction. The purpose is to compare our results with previous work. Nurhaida, et.al [7] discovered that using single GLCM feature with raw image input gave the best accuracy for batik motif classification. However, we will use the feature for our Batik Motif retrieval system task. Figure 4 depicts the framework of the proposed approach for batik motif retrieval system, which mainly includes of feature extraction process, data normalization, fusion at the feature level, and the measurement of similarity matching between query images against images in the database. The framework of feature extraction fusion is shown in Figure 4. 


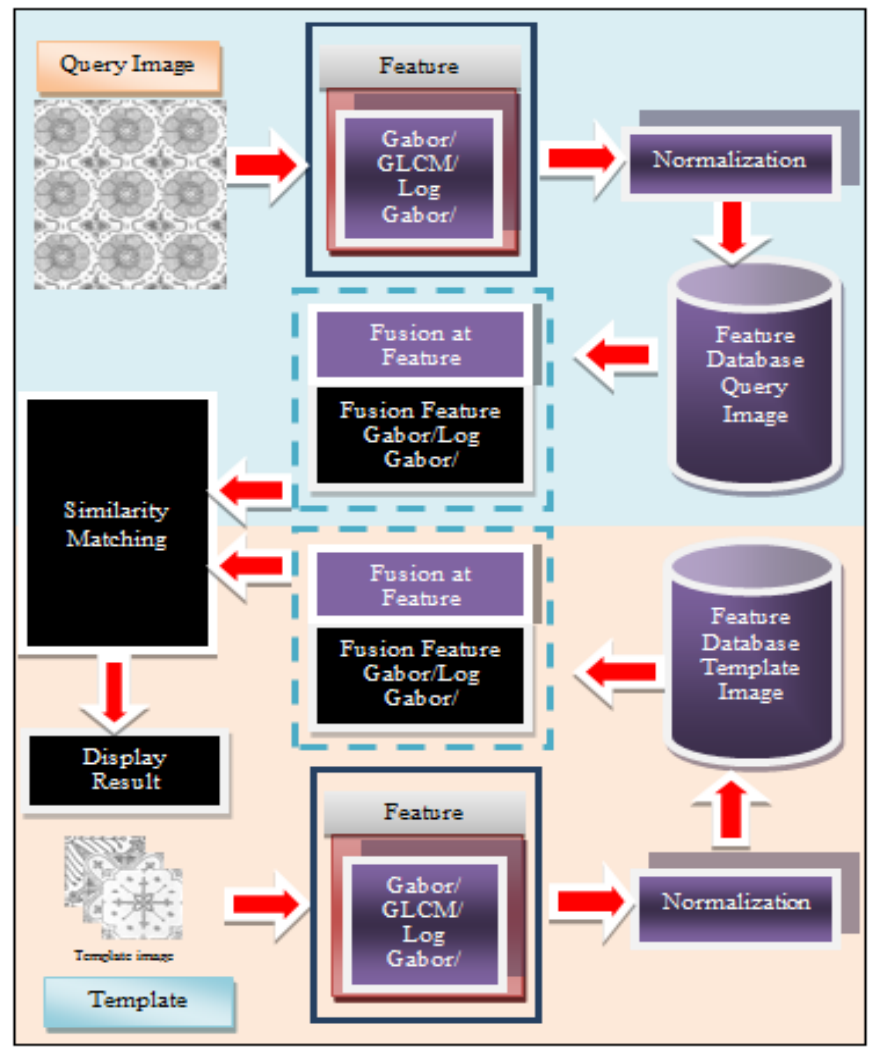

Figure 4. Framework of feature extraction fusion for Batik Motif Retrieval System

\section{EXPERIMENTAL DESIGN}

The dataset consists of 210 templates ( $150 \times 150$ pixels) motifs of batik images from 5 classes of geometric pattern. The images were firstly grouped into 5 classes based on the nature batik motifs. The 5 classes are ceplok, kawung, lereng, nitik, and parang. These templates were cropped into primitive patterns. The query dataset is generated from each template based on translation, scale, and rotation. The total number of generating query images is 3,165 in JPG format and they have a size of $450 \times 450$ pixels. The query images are generated based on the relevant template with a variation of translation, scale, and rotation. There is a number of templates in each query image ranging from 1 to 9 objects. Examples are shown in Figure 5.

In this research, we use Gabor filter, GLCM, Log Gabor filter, and Local Binary Pattern as texture features. For the Gabor filter bank, we choose 6 values of orientation: $0^{\circ}, 30^{\circ}, 60^{\circ}, 90^{\circ}, 120^{\circ}$, and $150^{\circ}$ and 4 frequencies are selected: 22.63, 11.31, 5.66, and 2.83 following [14], [29]. GLCM was computed over distance $\mathrm{d}=1$ and 4 directions $\left(0^{\circ}, 45^{\circ}, 90^{\circ}\right.$, and $\left.135^{\circ}\right)$. For Log Gabor, following [16], we choose four numbers of wavelet scales and six numbers of filter orientations. Local Binary Pattern (LBP) features are extracted using invariant parameter set to 1 which means every LBP label is computed using the minimal chain by rotating neighboring pixels.

In order to get the best retrieval result, the two types of dataset used as a query are explained below:

Dataset 1 : Image dataset which contained one basic motif object template from a particular class.

Dataset 2 : Image dataset which contained multiple objects

In this research we attempt three scenarios as follows:

Scenario 1 : One image containing one object is selected as a query image, while the rest images in dataset 1 are used as template images.

Scenario 2 : One image containing multiple objects is selected from the dataset 2, while the images in dataset 1 are used as template images.

Scenario 3 : One image containing multiple objects is selected from the dataset 2, while the rest image in dataset 2 will are as template images.

where:

Template images : images in database

Query images : images referring to user's task of searching for batik motif objects that are relevant to a query within images in database 


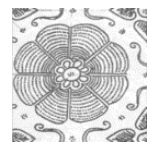

(a) Template Image 002-CPK003-Blibar11.png with Scale 1.0.

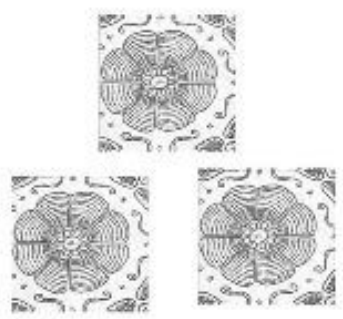

(b) Query Image with 3 Objects, Scale 0.5 and Rotation 0 Degree with Random Position

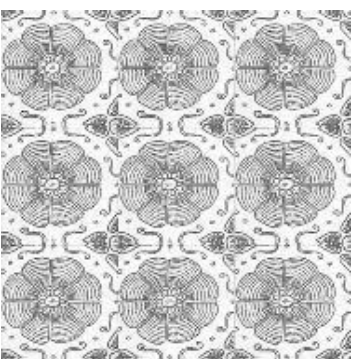

(c) Query data with 9 objects, Scale 0.5 and Rotation 0 Degree with Regular Pattern

Figure 5. Experiment Dataset Generated from Template Image

Each image in a dataset is used as a query image and template image alternately. The purpose of these scenarios is to find out whether the feature fusion will improve retrieval rate if the number of motif objects is added to template and query data incrementally. Furthermore, we investigate whether the system can discover the primitive motif that is contained in query image against template dataset. We first represent the images in the grey scale and then employ the feature extraction methods to gain the feature vector for each image. In particular, the image retrieval process is based on fusion process which utilized features resulted from feature extraction processes respectively. The feature vector is achieved as follows:

$$
F=\left\{f_{m_{1}}, f_{m_{2}}, f_{m_{3}}, f_{m_{4}}\right\}=\left\{\left\{f_{1,1}, f_{1,2}, \ldots, f_{1, n}\right\},\left\{f_{2,1}, f_{2,2}, \ldots, f_{2, n}\right\},\left\{f_{3,1}, f_{3,2}, \ldots, f_{3, n}\right\},\left\{f_{4,1}, f_{4,2}, \ldots, f_{4, n}\right\}\right\}
$$

where:

$f_{m}=$ feature vector resulted from each feature extraction method

Each type of features represents some image characteristic so that the fusion process will integrate image characteristics to fulfill image retrieval. Specifically, the feature vector is represented as a single vector. Therefore, we apply the normalization process which transforms all features into a comparable range of values. In other words, this normalization process changes the range of pixel intensity values between $[0-1]$. The query dataset and template dataset from the databases are used by the system to obtain the best 1, 3, 5, 7, 42 and 632 matched images ordered by their similarity, i.e. Euclidean distance. The Top-42 and Top-632 are the average number of template images and query images which distributed in each class consecutively. The aim is to achieve the best performance of the system that can retrieve up to those numbers of images. The similarity calculation of a single feature vector is as follows:

1) Calculate the average value of the feature vector $\mu_{f m}$ and variance $\sigma_{f m}$

2) Calculate Euclidian distance $\operatorname{Dist}(i, j)$ where $(i \neq j)$ between query image and template images in database

$$
\operatorname{Dist}(i, j)=\sqrt{\sum_{k=1}^{m}\left(r_{i k}-r_{j k}\right)^{2}}
$$

where:

$r=$ corresponding descriptor of feature $f_{m}$

3) For given query image $q$, calculate Euclidian distance with all image in template database

$$
\operatorname{Dist}(1, q), \operatorname{Dist}(2, q), \operatorname{Dist}(3, q), \ldots \operatorname{Dist}\left(m_{\text {total }}, q\right)
$$

where:

$m_{\text {total }}=$ total template images in the database

The performance of successful retrieval image is evaluated by the two measures, precision, and recall. 


\section{RESULT AND ANALYSIS}

Table 1 compares the precision and recall results obtained from the system on top- $N$ similar images from each class. Based on the experimental results using scenario 1, all single-feature and feature fusion methods are able to retrieve the same image from the image database. The fusion of Gabor, GLCM, LBP, and Log Gabor features gained the highest retrieval performance for the top 3 retrieval images, whereas the fusion of Gabor, Log Gabor, and GLCM features achieves the highest value for the top 5, 7, and 42. The best precision is $73.93 \%$ for the top 3 and the best recall is $32.53 \%$ for top 42 . The best precision and recall using a single feature is obtained using Log Gabor fea- ture with precision of $72.83 \%$ for the top 3 and recall of $31.88 \%$ for the top 42 .

Table 1. Precision and Recall of the Batik Motif Retrieval System for Template to Template Scenario 1

\begin{tabular}{|c|c|c|c|c|c|c|c|c|c|c|}
\hline \multirow{3}{*}{ Feature Extraction Method } & \multicolumn{10}{|c|}{ TOP } \\
\hline & \multicolumn{2}{|c|}{1} & \multicolumn{2}{|c|}{3} & \multicolumn{2}{|c|}{5} & \multicolumn{2}{|c|}{7} & \multicolumn{2}{|c|}{42} \\
\hline & Prec & Rec & Prec & Rec & Prec & Rec & Prec & Rec & Prec & $\operatorname{Rec}$ \\
\hline Gabor & $100 \%$ & $2.37 \%$ & $69.98 \%$ & $4.53 \%$ & $59.05 \%$ & $6.02 \%$ & $54.16 \%$ & $7.48 \%$ & $35.47 \%$ & $26.23 \%$ \\
\hline GLCM & $100 \%$ & $2.37 \%$ & $58.61 \%$ & $3.90 \%$ & $48.91 \%$ & $5.21 \%$ & $44.41 \%$ & $6.41 \%$ & $34.04 \%$ & $26.62 \%$ \\
\hline LBP & $100 \%$ & $2.37 \%$ & $57.50 \%$ & $3.55 \%$ & $48.34 \%$ & $4.86 \%$ & $42.99 \%$ & $5.82 \%$ & $31.79 \%$ & $22.86 \%$ \\
\hline Log-Gabor & $100 \%$ & $2.37 \%$ & $72.83 \%$ & $4.50 \%$ & $63.51 \%$ & $6.33 \%$ & $58.90 \%$ & $7.94 \%$ & $43.55 \%$ & $31.88 \%$ \\
\hline Gabor GLCM & $100 \%$ & $2.37 \%$ & $65.56 \%$ & $4.30 \%$ & $55.45 \%$ & $5.84 \%$ & $50.64 \%$ & $7.24 \%$ & $36.40 \%$ & $28.15 \%$ \\
\hline Gabor LBP & $100 \%$ & $2.37 \%$ & $64.61 \%$ & $4.16 \%$ & $55.17 \%$ & $5.64 \%$ & $50.85 \%$ & $7.01 \%$ & $35.14 \%$ & $26.11 \%$ \\
\hline Gabor Log-Gabor & $100 \%$ & $2.37 \%$ & $72.83 \%$ & $4.52 \%$ & $64.27 \%$ & $6.45 \%$ & $59.38 \%$ & $8.09 \%$ & $44.01 \%$ & $32.48 \%$ \\
\hline GLCM LBP & $100 \%$ & $2.37 \%$ & $60.66 \%$ & $3.99 \%$ & $52.23 \%$ & $5.41 \%$ & $47.12 \%$ & $6.63 \%$ & $34.18 \%$ & $26.21 \%$ \\
\hline GLCM Log-Gabor & $100 \%$ & $2.37 \%$ & $72.99 \%$ & $4.53 \%$ & $63.41 \%$ & $6.36 \%$ & $58.36 \%$ & $7.97 \%$ & $43.42 \%$ & $32.19 \%$ \\
\hline LBP Log-Gabor & $100 \%$ & $2.37 \%$ & $73.46 \%$ & $4.51 \%$ & $63.89 \%$ & $6.30 \%$ & $59.44 \%$ & $8.04 \%$ & $43.75 \%$ & $31.98 \%$ \\
\hline Gabor GLCM LBP & $100 \%$ & $2.37 \%$ & $66.35 \%$ & $4.33 \%$ & $55.55 \%$ & $5.78 \%$ & $50.98 \%$ & $7.29 \%$ & $36.77 \%$ & $28.23 \%$ \\
\hline Gabor GLCM Log-Gabor & $100 \%$ & $2.37 \%$ & $73.14 \%$ & $4.57 \%$ & $63.60 \%$ & $6.42 \%$ & $58.97 \%$ & $8.07 \%$ & $43.55 \%$ & $32.47 \%$ \\
\hline Gabor LBP Log-Gabor & $100 \%$ & $2.37 \%$ & $73.14 \%$ & $4.57 \%$ & $64.93 \%$ & $6.49 \%$ & $60.19 \%$ & $8.21 \%$ & $44.18 \%$ & $32.53 \%$ \\
\hline GLCM LBP Log-Gabor & $100 \%$ & $2.37 \%$ & $73.46 \%$ & $4.56 \%$ & $63.60 \%$ & $6.37 \%$ & $58.90 \%$ & $8.08 \%$ & $43.47 \%$ & $32.08 \%$ \\
\hline Gabor GLCM LBP Log-Gabor & $100 \%$ & $2.37 \%$ & $73.93 \%$ & $4.61 \%$ & $63.98 \%$ & $6.46 \%$ & $59.24 \%$ & $8.14 \%$ & $43.73 \%$ & $32.48 \%$ \\
\hline
\end{tabular}

From Figure 6, it is apparent that the first rank of the retrieved images from the template database is exactly the same image as a query image. As seen in Table 2, the feature fusion performance outperforms single-feature performance. Feature fusion from GLCM and Log Gabor achieves the best precision of $54.68 \%, 46.15 \%, 43.71 \%$ for top 1,3 , and 5, respectively. However, retrieval of the image for the top 42 obtains from feature fusion of LBP and Log Gabor at 36.40\%. Furthermore, retrieval rate from feature fusion outperforms the best single feature performance from Log Gabor feature which shows precision at 53.61\%, $46.04 \%, 43.45 \%$, and $36.08 \%$ for top $1,3,5$, and 42 , respectively. In this scenario, the results of recall are significantly lower than recall values from scenario 1 . It is due to insufficient information located in template dataset compared to the greater numbers of motif contained in the query image dataset. It means the query images have more complicated characteristic. Therefore, it is hard to find the relevant image in the template database. The retrieved images based on scenario 2 is shown in Figure 7.

Table 2. Precision and recall of the batik motif retrieval system for query to template scenario 2

\begin{tabular}{|c|c|c|c|c|c|c|c|c|c|c|}
\hline \multirow{3}{*}{ Feature Extraction Method } & \multicolumn{10}{|c|}{ TOP } \\
\hline & \multicolumn{2}{|c|}{1} & \multicolumn{2}{|c|}{3} & \multicolumn{2}{|c|}{5} & \multicolumn{2}{|c|}{7} & \multicolumn{2}{|c|}{42} \\
\hline & Prec & $\operatorname{Rec}$ & Prec & Rec & Prec & $\operatorname{Rec}$ & Prec & $\operatorname{Rec}$ & Prec & Rec \\
\hline Gabor & $29.84 \%$ & $0.61 \%$ & $29.33 \%$ & $1.81 \%$ & $29.25 \%$ & $2.96 \%$ & $29.28 \%$ & $4.12 \%$ & $28.95 \%$ & $21.93 \%$ \\
\hline GLCM & $32.59 \%$ & $0.61 \%$ & $31.46 \%$ & $1.78 \%$ & $31.19 \%$ & $2.96 \%$ & $30.37 \%$ & $4.07 \%$ & $28.91 \%$ & $22.39 \%$ \\
\hline LBP & $18.43 \%$ & $0.45 \%$ & $15.00 \%$ & $1.38 \%$ & $21.80 \%$ & $2.32 \%$ & $27.08 \%$ & $3.25 \%$ & $30.56 \%$ & $19.91 \%$ \\
\hline Log-Gabor & $53.51 \%$ & $1.15 \%$ & $46.04 \%$ & $2.76 \%$ & $43.45 \%$ & $4.25 \%$ & $41.57 \%$ & $5.55 \%$ & $36.08 \%$ & $25.24 \%$ \\
\hline Gabor GLCM & $32.24 \%$ & $0.65 \%$ & $31.23 \%$ & $1.99 \%$ & $30.42 \%$ & $3.18 \%$ & $29.96 \%$ & $4.43 \%$ & $28.62 \%$ & $23.17 \%$ \\
\hline Gabor LBP & $20.13 \%$ & $0.51 \%$ & $30.01 \%$ & $1.56 \%$ & $33.12 \%$ & $2.57 \%$ & $33.61 \%$ & $3.56 \%$ & $32.87 \%$ & $20.73 \%$ \\
\hline Gabor Log-Gabor & $52.15 \%$ & $1.14 \%$ & $44.99 \%$ & $2.76 \%$ & $42.49 \%$ & $4.24 \%$ & $40.85 \%$ & $5.52 \%$ & $35.81 \%$ & $25.49 \%$ \\
\hline GLCM LBP & $31.45 \%$ & $0.59 \%$ & $33.02 \%$ & $1.69 \%$ & $34.06 \%$ & $2.75 \%$ & $32.27 \%$ & $3.76 \%$ & $30.25 \%$ & $20.65 \%$ \\
\hline GLCM Log-Gabor & $54.68 \%$ & $1.18 \%$ & $46.15 \%$ & $2.80 \%$ & $43.71 \%$ & $4.32 \%$ & $41.27 \%$ & $5.61 \%$ & $35.79 \%$ & $25.33 \%$ \\
\hline LBP Log-Gabor & $48.96 \%$ & $0.95 \%$ & $44.23 \%$ & $2.44 \%$ & $42.66 \%$ & $3.87 \%$ & $41.56 \%$ & $5.15 \%$ & $36.40 \%$ & $24.90 \%$ \\
\hline Gabor GLCM LBP & $29.90 \%$ & $0.61 \%$ & $32.80 \%$ & $1.77 \%$ & $33.40 \%$ & $2.86 \%$ & $33.46 \%$ & $3.89 \%$ & $32.32 \%$ & $21.62 \%$ \\
\hline Gabor GLCM Log-Gabor & $53.41 \%$ & $1.17 \%$ & $45.15 \%$ & $2.77 \%$ & $42.54 \%$ & $4.31 \%$ & $40.62 \%$ & $5.60 \%$ & $35.64 \%$ & $25.58 \%$ \\
\hline Gabor LBP Log-Gabor & $48.70 \%$ & $0.93 \%$ & $44.58 \%$ & $2.47 \%$ & $42.84 \%$ & $3.92 \%$ & $41.58 \%$ & $5.15 \%$ & $36.22 \%$ & $25.08 \%$ \\
\hline GLCM LBP Log-Gabor & $51.14 \%$ & $1.00 \%$ & $45.00 \%$ & $2.55 \%$ & $43.02 \%$ & $3.95 \%$ & $41.11 \%$ & $5.18 \%$ & $36.18 \%$ & $24.92 \%$ \\
\hline Gabor GLCM LBP Log-Gabor & $49.94 \%$ & $0.99 \%$ & $45.11 \%$ & $2.55 \%$ & $42.52 \%$ & $3.96 \%$ & $41.26 \%$ & $5.24 \%$ & $36.12 \%$ & $25.13 \%$ \\
\hline
\end{tabular}



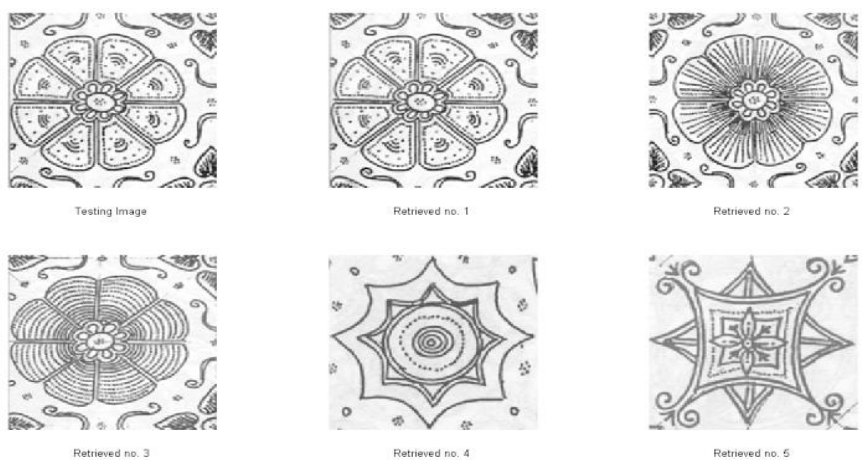

Figure 6. The retrieved images at top 5 based on 1 motif template as a query image against to template images in database (scenario 1)
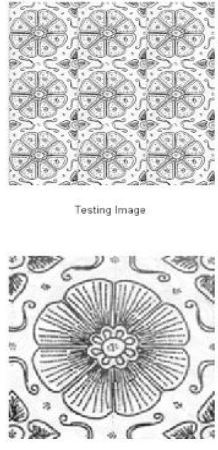

Retrieved no.
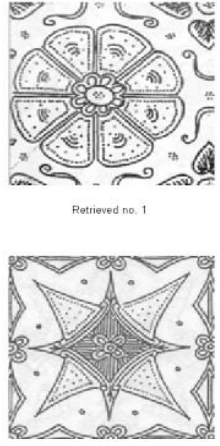

Retrieved no.
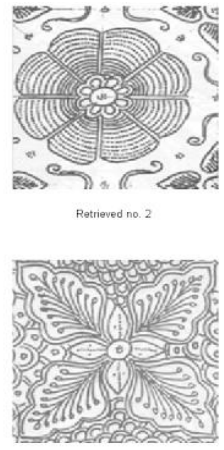

Retrieved no. 5

Figure 7. The retrieved images based on query image at top 5 that contained more than 1 motif as a query image against to template images in the database (scenario 2)

Table 3 illustrates some characteristics of image retrieval results from the system for scenario 3 . A positive correlation was found between an increase in the number of motifs in query images and template image in precision. Furthermore, it can be seen from Table 3 that feature fusion outperforms single features for precision at 3,5 , and 7 .

The best precision is $84.36 \%$ achieved from feature fusion of Gabor, GLCM, and Log Gabor for top 3; while the single feature achieves $83.28 \%$ from Log Gabor as the best. Meanwhile, recall gets $0.38 \%$, $0.56 \%, 0.71 \%$, and $23.88 \%$ for top $3,5,7$, and 632 consecutively. These results are likely to be related to adequate information found in both query image and image in the database to find the similar image. The retrieved images based on scenario 3 can be seen in Figure 8.

Table 3. Precision and recall of the batik motif retrieval system for query to query (scenario 3 )

\begin{tabular}{|c|c|c|c|c|c|c|c|c|c|c|}
\hline \multirow{3}{*}{ Feature Extraction Method } & \multicolumn{10}{|c|}{ TOP } \\
\hline & \multicolumn{2}{|c|}{1} & \multicolumn{2}{|c|}{3} & \multicolumn{2}{|c|}{5} & \multicolumn{2}{|l|}{7} & \multicolumn{2}{|c|}{632 (average) } \\
\hline & Prec & $\operatorname{Rec}$ & Prec & Rec & Prec & Rec & Prec & $\operatorname{Rec}$ & Prec & Rec \\
\hline Gabor & $100 \%$ & $2.37 \%$ & $74.94 \%$ & $0.33 \%$ & $64.67 \%$ & $0.46 \%$ & $59.02 \%$ & $0.58 \%$ & $30.38 \%$ & $21.36 \%$ \\
\hline GLCM & $100 \%$ & $2.37 \%$ & $67.80 \%$ & $0.30 \%$ & $58.09 \%$ & $0.41 \%$ & $51.87 \%$ & $0.50 \%$ & $29.73 \%$ & $21.44 \%$ \\
\hline LBP & $100 \%$ & $2.37 \%$ & $59.06 \%$ & $0.25 \%$ & $48.93 \%$ & $0.33 \%$ & $44.51 \%$ & $0.41 \%$ & $28.83 \%$ & $19.99 \%$ \\
\hline Log-Gabor & $100 \%$ & $2.37 \%$ & $83.28 \%$ & $0.38 \%$ & $75.34 \%$ & $0.55 \%$ & $69.56 \%$ & $0.69 \%$ & $35.36 \%$ & $23.79 \%$ \\
\hline Gabor GLCM & $100 \%$ & $2.37 \%$ & $75.00 \%$ & $0.34 \%$ & $64.85 \%$ & $0.47 \%$ & $58.38 \%$ & $0.58 \%$ & $30.20 \%$ & $21.92 \%$ \\
\hline Gabor LBP & $100 \%$ & $2.37 \%$ & $70.66 \%$ & $0.31 \%$ & $59.72 \%$ & $0.41 \%$ & $54.20 \%$ & $0.51 \%$ & $29.68 \%$ & $20.83 \%$ \\
\hline Gabor Log-Gabor & $100 \%$ & $2.37 \%$ & $83.87 \%$ & $0.38 \%$ & $75.80 \%$ & $0.56 \%$ & $70.07 \%$ & $0.70 \%$ & $35.30 \%$ & $23.88 \%$ \\
\hline GLCM LBP & $100 \%$ & $2.37 \%$ & $64.36 \%$ & $0.28 \%$ & $54.00 \%$ & $0.38 \%$ & $48.98 \%$ & $0.47 \%$ & $29.14 \%$ & $21.02 \%$ \\
\hline GLCM Log-Gabor & $100 \%$ & $2.37 \%$ & $83.90 \%$ & $0.38 \%$ & $76.00 \%$ & $0.55 \%$ & $70.33 \%$ & $0.69 \%$ & $35.20 \%$ & $23.82 \%$ \\
\hline LBP Log-Gabor & $100 \%$ & $2.37 \%$ & $82.49 \%$ & $0.37 \%$ & $73.84 \%$ & $0.53 \%$ & $68.21 \%$ & $0.67 \%$ & $34.05 \%$ & $23.20 \%$ \\
\hline Gabor GLCM LBP & $100 \%$ & $2.37 \%$ & $69.90 \%$ & $0.31 \%$ & $59.06 \%$ & $0.42 \%$ & $53.81 \%$ & $0.52 \%$ & $29.44 \%$ & $21.29 \%$ \\
\hline Gabor GLCM Log-Gabor & $100 \%$ & $2.37 \%$ & $84.36 \%$ & $0.38 \%$ & $76.16 \%$ & $0.56 \%$ & $70.81 \%$ & $0.70 \%$ & $35.07 \%$ & $23.88 \%$ \\
\hline Gabor LBP Log-Gabor & $100 \%$ & $2.37 \%$ & $83.07 \%$ & $0.37 \%$ & $74.75 \%$ & $0.54 \%$ & $68.94 \%$ & $0.68 \%$ & $34.07 \%$ & $23.29 \%$ \\
\hline GLCM LBP Log-Gabor & $100 \%$ & $2.37 \%$ & $82.99 \%$ & $0.37 \%$ & $74.94 \%$ & $0.54 \%$ & $69.17 \%$ & $0.68 \%$ & $33.85 \%$ & $23.22 \%$ \\
\hline Gabor GLCM LBP Log-Gabor & $100 \%$ & $2.37 \%$ & $83.54 \%$ & $0.38 \%$ & $75.43 \%$ & $0.55 \%$ & $69.67 \%$ & $0.69 \%$ & $33.82 \%$ & $23.29 \%$ \\
\hline
\end{tabular}

Texture Fusion for Batik Motif Retrieval System (Ida Nurhaida) 


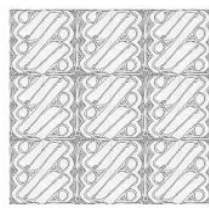

Testing Image

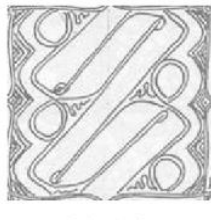

Retrieved no. 3

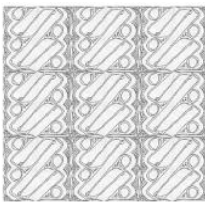

Retivered no.

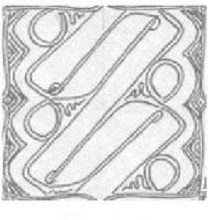

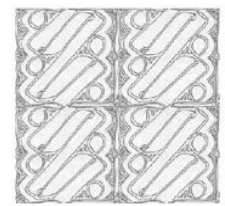

Retrieved no. 2

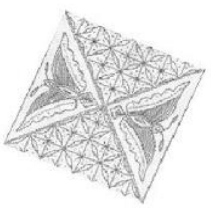

Figure 8 . The retrieved images based on query image at top 5 that contained more than 1 motifs against to query images in image database (scenario 3 )

Batik motifs have complex behavior like natural images. Our datasets contain similarity patterns, periodical motifs, and random features within images where motifs look like unstructured noise (see Figure 9). The fusion process integrates the nature of each feature to increase the retrieval rate. The most significant finding is that Log Gabor features outperformed other methods, not only as a single feature but also as part of feature fusion. Gabor filters are able to describe texture information in low and medium frequencies, but the filters become inconsistent in measurement for the high frequency information [14], thus becoming more sensitive to noise. The high frequency filter has larger spatial-frequency bandwidth which covers relatively more energy from the noise that is evenly distributed in the spatial domain. This distortion may cause poor accuracy for pattern retrieval. Log Gabor can cover a large frequency space while still maintaining a zero DC component in the even symmetric filter [28]. Log Gabor filter is preferred if the bandwidth increases with high frequency. The information will be equally spread by Log Gabor across the scale. GLCM is not as sensitive to noise as Gabor Filter. However, GLCM is not proper for estimating lower frequency features [30]. The fusion of low and medium frequency Gabor filter features, Log Gabor features and high frequency from GLCM features is the best combination to generate an improved feature set.

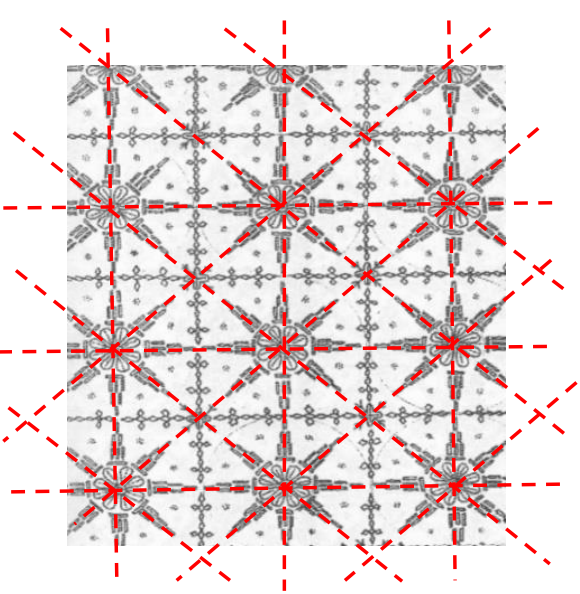

(a) Nitik Sekar Rumpuk

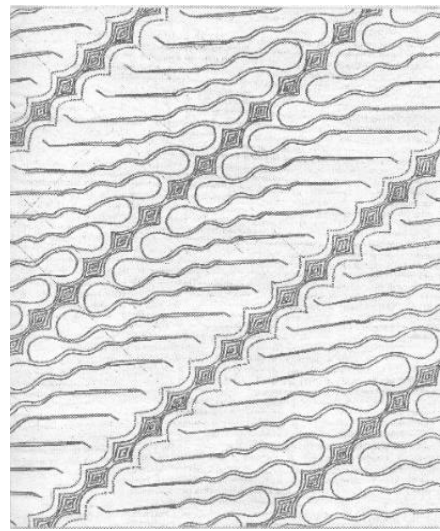

(b) Parang Curiga

Figure 9. The geometric patterns are recognizable due to the symmetry and repetition in horizontal, vertical, and diagonal directions that form angles between shapes. Nitik motif has classified into the geometric design which created with small dots and dashes imitating the original woven fabric. Parang Curiga motif takes the form of a gently curved design in a powerful rhythm

Conversely, Local Binary Patterns have the lowest performance in the case of Batik Motif Retrieval. The LBP depicts texture image from small symmetric neighborhoods using local structure. Thus, it may not 
capture key texture characteristics. The binary information will be preserved while the local intensity difference is ignored. A larger region size will result in a decreased recognition rate because of the loss of spatial information. The pairs of neighbors are compared only if their connecting lines pass through the center pixel. LBP may not cover for the multi-resolution technique since the correlation of patterns under different scales is also discarded.

Based on the result above, we can see that in all of the experiments our method by fusing several texture features gave better performance than single feature GLCM used in previous work [7]. The comparison between our work and previous work can be seen in Figure 10. We sample the best Top-3 results from each experiment and compare it with previous work. Overall, our method improves the previous work result by $15.32 \%, 14.69 \%$, and $8.20 \%$ for scenario 1,2 , and 3 (Top-3) respectively.

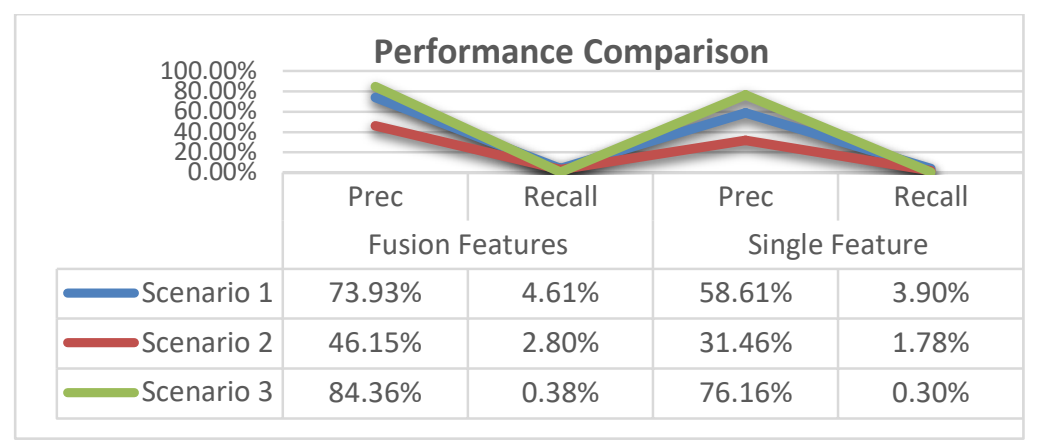

Figure 10. Performance comparison with previous work [7]

\section{CONCLUSION}

A systematic investigation was conducted into the impact of image texture features on a batik motif retrieval system. Feature fusion was attempted to improve the retrieval rate. A large amount of experimental work has been carried out. The following conclusions can be drawn:

a. If only using an individual feature, log-Gabor should be considered since it provides a relatively high retrieval rate.

b. Feature fusion can significantly improve the retrieval rate when combining log-Gabor, GLCM, and Gabor features in the feature space.

c. LBP shows less contribution to batik motif retrieval.

d. The proposed approach is best used in the scenario where a query image containing multiple basic motif objects is applied to a dataset in which retrieved images also contain multiple motif objects.

e. The proposed method does not work well for a retrieval scenario where the query image contains multiple basic motif objects being applied to a dataset in which the retrieved images only contain one basic motif object.

f. The proposed method also works for a scenario where the query image contains only one basic motif object and the retrieved images are also contain one motif object.

It is important to fuse features that complement information naturally in the feature space. In this study, the careful selection of log-Gabor features, GLCM, and Gabor features gives a full cover of spatial frequency spectrum for batik motif retrieval.

\section{ACKNOWLEDGEMENTS}

This work has been sponsored by the grant Ministry of Research, Technology and Higher Education of the Republic of Indonesia, 2016

\section{REFERENCES}

[1] S. S. Samsi, "Techniques, Motifs, Patterns Batik Yogya and Solo," Titian Foundation, 2011.

[2] H. S. Doellah, "Batik: The Impact of Time and Environment," Danar Hadi Solo, 2003.

[3] J. Achjadi, "The Glory of Batik: The Danar Hadi Collection, 1st ed," Central Java, PT. Batik Danar Hadi, 2011.

[4] A. K. Jain, et al., "Content-Based Image Retrieval: an Application to Tattoo Images," in ICIP'09 Proceedings of the 16th IEEE international conference on Image processing, pp. 2745-2748, 2009.

[5] M. S. Lew, "Content-based Multimedia Information Retrieval: State of the Art and Challenges," ACM Trans. Multimed. Comput. Commun. Appl., 2006. 
[6] S. Aksoy and R. M. Haralick, "A Classification Framework for Content-Based Image Retrieval," in International Conference on Pattern Recognition - ICPR, Seattle, vol. 2, pp. 503-506, 2002.

[7] I. Nurhaida, et al., "Performance Comparison Analysis Features Extraction Methods for Batik Recognition," in International Conference on Advanced Computer Science and Information Systems, Depok, 2012.

[8] A. H. Rangkuti, et al., "Batik Image Retrieval Based on Similarity of Shape and Texture Characteristics," in International Conference on Advanced Computer Science and Information Systems, Depok, pp. 978-979, 2012.

[9] A. E. Minarno, et al., "Batik image retrieval based on enhanced micro-structure descriptor," 2014 Asia-Pacific Conf. Comput. Aided Syst. Eng. (APCASE), Bali, vol. 1, pp. 65-70, 2014.

[10] H. Rangkuti, et al., "Content Based Batik Image Retrieval," J. Comput. Sci., vol/issue: 10(6), pp. 925-934, 2014.

[11] M. Bartels and H. Wei, "Unsupervised Segmentation Using Gabor Wavelets and Statistical Features in LIDAR Data Analysis," in 18th International Conference on Pattern Recognition (ICPR'06), pp. 667-670, 2006.

[12] A. K. Jain and F. Farrokhnia, "Unsupervised Texture Segmentation Using Gabor Filters," in IEEE International Conference onSystems, Man and Cybernetics, pp. 14-19, 1990.

[13] M. Mirmehdi, et al., "Handbook of Texture Analysis," London, World Scientific Publishing, 2008.

[14] D. A. Clausi and H. Deng, "Design-Based Texture Feature Fusion Using Gabor Filters and Co-Occurrence Probabilities," Image Process. IEEE Trans., vol/issue: 14(7), pp. 925-936, 2005.

[15] A. Fahrurozi, et al., "Wood Classification Based on Edge Detections and Texture Features Selection," Int. J. Electr. Comput. Eng., vol/issue: 6(5), pp. 2167-2175, 2016.

[16] D. J. Field, "Relations between the statistics of natural images and the response properties of cortical cells," J. Opt. Soc. Am. A Opt. Image Sci., vol/issue: 4(12), 1987.

[17] R. Nava, et al., "Texture Image Retrieval Based on Log-Gabor Features,” in CIARP, pp. 414-421, 2012.

[18] S. M. Lajevardi and M. Lech, "Facial Expression Recognition Using Neural Networks and Log-Gabor Filters," 2008 Digit. Image Comput. Tech. Appl., pp. 77-83, 2008.

[19] B. Gosselin, "Character Segmentation-by-Recognition Using Log-Gabor Filters," in Pattern Recognition 18th International Conference on, pp. 901-904, 2006.

[20] R. Nava, et al., "A Comparison Study of Gabor and Log-Gabor Wavelets for Texture Segmentation," in International Symposium on Image and Signal Processing and Analysis, pp. 189-194, 2011.

[21] S. Yasmin and M. M. Rana, "Performance Study of Soft Local Binary Pattern over Local Binary Pattern under Noisy Images,” Int. J. Electr. Comput. Eng., vol/issue: 6(3), pp. 1161, 2016.

[22] M. Pietikäinen, et al., "Computer Vision Using Local Binary Patterns," London, Springer London, vol. 40, 2011.

[23] N. Liu, et al., "Multimodal recognition of visual concepts using histograms of textual concepts and selective weighted late fusion scheme," Comput. Vis. Image Underst., vol/issue: 117(5), pp. 493-512, 2013.

[24] Z. H. Huang, et al., "Face recognition based on pixel-level and feature-level fusion of the top-level's wavelet subbands," Inf. Fusion, vol. 22, pp. 95-104, 2015.

[25] A. Jain, et al., "Score normalization in multimodal biometric systems $\square$, , J. Pattern Recognit., vol. 38, pp. 2270 $2285,2005$.

[26] M. F. Demirci, et al., "Efficient many-to-many feature matching under the 11 norm," Comput. Vis. Image Underst., vol/issue: 115(7), pp. 976-983, 2011.

[27] D. Hou, et al., "A Content-Based Retrieval Algorithm for Document Image Database," in 2010 International Conference on Multimedia Technology, pp. 1-5, 2010.

[28] R. Hu and J. Collomosse, "A performance evaluation of gradient field HOG descriptor for sketch based image retrieval," Comput. Vis. Image Underst., 2013.

[29] D. A. Clausi and M. E. Jernigan, "Designing Gabor Filters for optimal texture separability," Pattern Recognit. J., vol. 33, pp. 1835-1849, 2000.

[30] D. A. Clausi and H. Deng, "Fusion of Gabor Filter and Co-occurrence Probability Features for Texture Recognition Co-occurrence Probabilities," IEEE Trans. Image Process., 2005.

\section{BIOGRAPHIES OF AUTHORS}

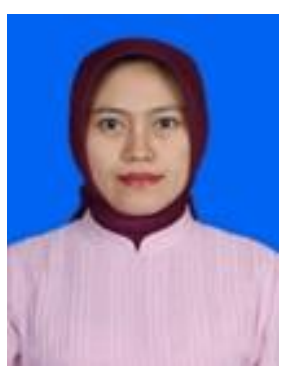

Ida Nurhaida achieved her undergraduate degree in Department of Electrical Engineering from Hasanuddin University, Makassar, Indonesia, in 2003. She is currently working toward as lecturer in Department of Informatics, Mercu Buana University, and Jakarta, Indonesia. She earned her Ph.D degree in Faculty of Computer Science from University of Indonesia, Depok, Indonesia, in 2010. Her current research activities are in image processing, pattern recognition and image retrieval system. 

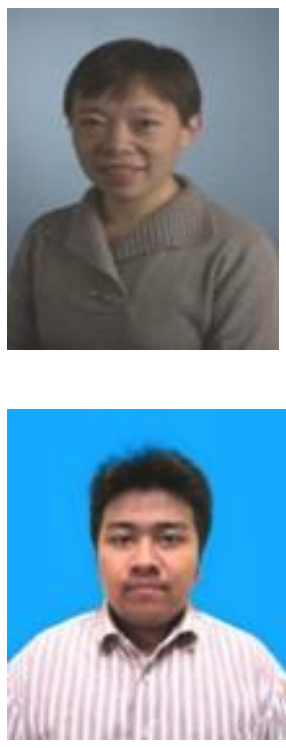

Remmy A. M. Zen was born in Jakarta, Indonesia in 1991. He received his Bachelor Degree in Computer Science from Universitas Indonesia in 2013 and Master Degree in Computer Science from Universitas Indonesia in 2014. He is currently working as a teaching assistant for undergraduate degree and also research assistant at Machine Learning and Computer Vision Lab at Faculty of Computer Science, Universitas Indonesia. His research interests includes Machine Learning, Image Processing, Computational Intelligence, Information Retrieval, and Software Technology.

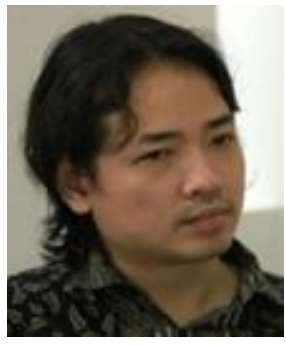

Ruli Manurung is a faculty member at Universitas Indonesia, where he currently serves as the Coordinator for the various computer sciences study programs. His research interests vary between natural language processing, computational creativity, and machine learning. He obtained his Ph.D from the University of Edinburgh in 2004 for his work on the automatic generation of poetry. He was a Research Fellow at the University of Edinburgh on an EPSRC research project that concerned the development of an innovative system that provided languageimpaired children with the ability to tell novel jokes.

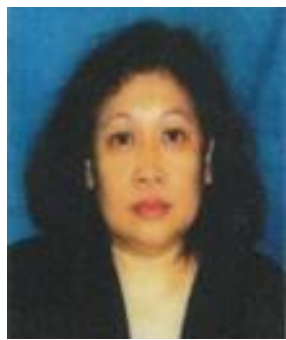

Aniati Murni Arymurthy is a Professor in Faculty of Computer Science, University of Indonesia. She graduated from Department of Electrical Engineering, University of Indonesia, and Jakarta, Indonesia. She earned her Master of Science in Department of Computer and Information Sciences, The Ohio State University (OSU), Columbus, Ohio, USA. She also holds Doktor from Department of Opto-Electronics and Laser Applications, University of Indonesia, Jakarta, Indonesia. Her main research activities are image processing and pattern recognition. 